\title{
Autophagy mediates free fatty acid effects on MDA-MB-231 cell proliferation, migration and invasion
}

\author{
JUAN WU ${ }^{1}$, QI WU ${ }^{1}$, JUAN-JUAN LI ${ }^{1}$, CHUANG CHEN $^{1}$, SI SUN $^{2}$, CHANG-HUA WANG $^{3}$ and SHENG-RONG SUN ${ }^{1}$ \\ Departments of ${ }^{1}$ Breast and Thyroid Surgery and ${ }^{2}$ Clinical Laboratory, Renmin Hospital of Wuhan University, \\ Wuhan, Hubei 430060; ${ }^{3}$ Department of Pathology and Pathophysiology, \\ Wuhan University School of Basal Medical School, Wuhan, Hubei 430071, P.R. China
}

Received April 8, 2016; Accepted June 6, 2017

DOI: $10.3892 / \mathrm{ol} .2017 .6807$

\begin{abstract}
Epidemiological and animal studies indicate an association between high levels of dietary fat intake and an increased risk of breast cancer. The multifaceted role of autophagy in cancer has been revealed in previous years. However, the mechanism of this role remains unknown. In the present study, the two most common free fatty acids, palmitate acid (PA) and oleic acid (OA), were used to determine the effect on human breast cancer MDA-MB-231 cells, and the possible role of autophagy was investigated by detecting light chain 3 (LC3)-II/I. Bafliomycin A1 was used to detect autophagy flux. High palmitate acid condition-induced MDA-MB-231 cell death and invasion were mitigated by 3-methyladenine pretreatment or transfection with shRNA against autophagy protein 5. By contrast, high oleic acid condition induced MDA-MB-231 cell proliferation, migration and invasion were mitigated using rapamycin. The present results suggest that autophagy has an important role in the effects of PA and OA on breast cancer growth and metastasis in vitro.
\end{abstract}

\section{Introduction}

Breast cancer is the second most common type of cancer globally. Although the majority of patients have a good outcome, the morbidity rate is increasing rapidly. In 2017, an estimated 252,710 females and 2,470 males will be diagnosed with

Correspondence to: Dr Chang-Hua Wang, Department of Pathology and Pathophysiology, Wuhan University School of Basal Medical School, 185 Donghu Road, Wuhan, Hubei 430071, P.R. China

E-mail: chwang0525@whu.edu.cn

Dr Sheng-Rong Sun, Department of Breast and Thyroid Surgery, Renmin Hospital of Wuhan University, 9 Zhang Zhi Dong Street, Wuchang, Wuhan, Hubei 430060, P.R. China

E-mail: sun137@sina.com

Key words: free fatty acid, palmitate acid, oleic acid, autophagy, $\mathrm{LC} 3$ invasive breast cancer, and 40,610 females and 460 males are expected to succumb to the disease (1). In China, female breast cancer was the most prevalent cancer in 2011, with the 5-year prevalence estimate reaching 1.02 million (2).

In the investigation of breast cancer, an excessive intake of dietary fat, obesity and diabetes have been found to be associated with cancer development (3). Free fatty acids (FFAs) act as substrates in energy metabolism and mediators in signal transduction, and are stored as lipid droplets (LDs) in hepatocytes and supply energy under poor conditions (4,5). A growing number of in vitro cell culture, animal cancer model and epidemiological studies, and clinical studies in human subjects, have provided evidence to support the effects of FFAs on cancer since almost 40 years ago (6-9). Unsaturated FFAs stimulated the proliferation of human breast cancer cells, whereas saturated FFAs inhibited cell proliferation and increased apoptosis (8). The high dietary intake of $n-3$ polyunsaturated fatty acids (PUFAs) shows a protective association with cancer risk (9); however, n-6 PUFAs are associated with an increased risk for the development of breast cancer $(6,10)$. The mechanism of PUFAs remains unclear.

Autophagy has gained attention recently as an essential contributor to human disease. The term 'autophagy' is derived from Greek, meaning 'self-eat'. As the only mechanism to degrade large structures such as organelles and protein aggregates, it has a double-edged role in human health and metabolism (11). In addition to its ability to promote cancer by allowing cells to survive under conditions of metabolic and genotoxic stress, excessive autophagy may paradoxically lead to cell death (12). Successful completion of the complex process of autophagy requires the coordinated function of a number of proteins and complexes at different steps, and tight regulation of each step by upstream modulators aids in fine-tuning the autophagic process $(13,14)$.

Autophagy is considered to promote cancer by allowing cells to survive under conditions of metabolic and genotoxic stress, and is additionally associated with cancer cell death induced by autophagic lysosome features (15). At present, no study has identified an association between autophagy and FFA-induced breast cancer cell metabolism. In the present study, we created high FFA condition by using two types of FFA to assess the effect of FFAs on human breast cancer MDA-MB-231 cells and identify a possible role of autophagy. 


\section{Materials and methods}

Cell culture and reagents. Human breast cancer MDA-MB-231 cells were cultured in high-glucose Dulbecco's modified Eagle's medium (DMEM; HyClone; GE Healthcare Life Sciences, Logan, UT, USA) containing $10 \%$ fetal bovine serum (FBS) (ScienCell Research Laboratories, Inc., Carlsbad, CA, USA) and $1 \%$ penicillin/streptomycin (Invitrogen; Thermo Fisher Scientific, Inc., Waltham, MA, USA) at $37^{\circ} \mathrm{C}$ in a humidified atmosphere containing $5 \% \mathrm{CO}_{2}$. Palmitate acid (PA) and oleic acid (OA) were purchased from Sigma-Aldrich (Merck KGaA, Darmstadt, Germany), and essentially fatty acid-free bovine serum albumin (BSA) was obtained from Roche Applied Science (Penzberg, Germany). The stock solutions of fatty acids bound to 5\% BSA were prepared as follows. The corresponding fatty acid was dissolved in a small amount of ethanol in a $37^{\circ} \mathrm{C}$ water bath, and then mixed with $5 \%$ fatty acid-free BSA to obtain a $10 \mathrm{mM}$ fatty acid stock solution. Subsequent to being adjusted to $\mathrm{pH} 7.4$, the solution was filtered through a $0.45 \mu \mathrm{m}$ filter. Finally, the solutions were subpackaged and stored at $-20^{\circ} \mathrm{C}$; equal ethanol and BSA were prepared as a control. The fatty acid stock solutions were then diluted in culture medium to obtain various concentrations of working solution, $0.25,0.50$ and $0.75 \mathrm{mmol} / \mathrm{l}$; the control was always selected with the maximum concentration of BSA (0.25\%). The antibody against LC3 (M152-3) was purchased from (Medical and Biological Laboratories, Nagoya, Japan), whereas the anti- $\beta$-actin antibody (cat. no. 37008H10D10), horse anti-mouse IgG (cat. no. 7076) and goat anti-rabbit IgG (cat. no. 7074) antibodies were purchased from Cell Signaling Technology, Inc. (Danvers, MA, USA). LC3 and $\beta$-actin were diluted in 5\% BSA with a ratio of 1:500, whereas the anti-mouse $\operatorname{IgG}$ and the anti-rabbit $\operatorname{IgG}$ were diluted with the ratio of 1:20,000 fresh before use. Bafliomycin A1 (BFA) and 3-methyladenine (3-MA) were purchased from Sigma-Aldrich (Merck KGaA). Rapamycin was purchased from MedChem Express (Monmouth Junction, NJ, USA).

Proliferation assay. The MTT assay was used to evaluate cell proliferation. MTT (Amresco, LLC, Solon, OH, USA) was dissolved in $\mathrm{ddH}_{2} \mathrm{O}$ at a concentration of $5 \mathrm{mg} / \mathrm{ml}$, filtered through a $0.22 \mu \mathrm{m}$ filter, and stored at $4^{\circ} \mathrm{C}$. Cells were seeded at a density of 5,000 cells/well in 96-well plate and cultured in stand medium at $37^{\circ} \mathrm{C}$ for at least $12 \mathrm{~h}$ until all cells were adhered. Then cells were treated with different concentrations of culture medium containing FFAs $(0.25,0.50$ and $0.75 \mathrm{mmol} / \mathrm{l}$ ) with $5 \% \mathrm{BSA}$ or FFA-free BSA for $24 \mathrm{~h}$. For the proliferation assay, $20 \mu \mathrm{l}$ MTT was added into each well. An ELISA plate reader (BioTek Instruments, Inc., Winooski, VT, USA) was used to measure the optical density at $490 \mathrm{~nm}$.

TUNEL assay. The TUNEL assay was performed using an In Situ Cell Death Detection kit (Roche Diagnostics, Basel, Switzerland), according to the manufacturer's instructions. Cells were seeded at a density of $2 \times 10^{5}$ cells/well in 24-well plate and cultured in DMEM without FBS at $37^{\circ} \mathrm{C}$ for at least $12 \mathrm{~h}$ until all cells were adhered. Subsequently, the cells were treated with various concentrations of culture medium containing FFAs $(0.25$ and $0.50 \mathrm{mmol} / \mathrm{l})$ with $5 \% \mathrm{BSA}$ or FFA-free BSA for $24 \mathrm{~h}$ at $37^{\circ} \mathrm{C}$. Cells were fixed with $4 \%$ paraformaldehyde for $1 \mathrm{~h}$ at room temperature. Then $0.2 \%$ TrionX-100 was used as a permeabilisation solution for $20 \mathrm{~min}$ at $4^{\circ} \mathrm{C}$ prior to TUNEL reagent (enzyme solution, label solution=1:9) and DAPI (100 $\mathrm{ng} / \mathrm{ml})$ were respectively used for $60 \mathrm{~min}$ and $10 \mathrm{~min}$ at $37^{\circ} \mathrm{C}$ to stain the nucleus. Finally, three fields of view were randomly selected and images (magnification, x400) were captured using the Olympus Fluo View FV1000 Confocal Microscope (Olympus, Tokyo, Japan).

Wound healing assay. MDA-MB-231 cells were grown to confluent monolayers on 6-well plates and a pipette tip $(200 \mu \mathrm{l})$ was used to create linear scratch wounds. Mitomycin C (Amresco, LLC) was used to inhibit cell proliferation. Wound images were captured using a digital camera mounted on a light microscope (magnification, x200). The wound gap widths were measured using ImageJ software (National Institutes of Health, Bethesda, MD, USA).

Transwell assay. The upper chamber of each $8.0-\mu \mathrm{m}$ pore size Transwell apparatus (Corning Inc., Corning, NY, USA) was coated with Matrigel (BD Biosciences, San Jose, CA, USA). MDA-MB-231 cells were added to the upper chamber at a density of $2 \times 10^{6}$ cells/ml (100 $\mu \mathrm{l} /$ chamber) and incubated for $24 \mathrm{~h}$, followed by removal of the cells that remained in the top chamber with cotton swabs. Cells that penetrated to the lower membrane surface were fixed in $4 \%$ paraformaldehyde, stained with crystal violet, and counted under a light microscope (magnification, $\mathrm{x} 400$ ).

shRNA transfection. The autophagy protein 5 (ATG5) shRNA and a non-specific shRNA (mock or control) were purchased from GeneChem, Inc. (Daejeon, Korea). According to the manufacturer's instructions, the transfections were performed at $\sim 60 \%$ confluency using Lipofectamine 2000 (Invitrogen; Thermo Fisher Scientific, Inc.). For each transfection reaction, $4 \mu \mathrm{g}$ shRNA was used for the preparation of the shRNA-transfection complexes at room temperature for $20 \mathrm{~min}$. The transfections were performed in 293T cells. Targeted MDA-MB-231 cell transfection was then performed using obtained virus fluid from 293T cells under the addition of $1 \mu \mathrm{g} / \mu \mathrm{l}$ polybrene for $24 \mathrm{~h}$. Subsequent to incubation, the transfection complexes were removed and replaced with their corresponding media with puromycin. The transfection efficiency was determined by western blotting. Successfully transfected MDA-MB-231 cells were used for subsequent experiments one week after amplification.

Western blotting. Cells were collected with lysis buffer (Cell Signaling Technology, Inc.) subsequent to being washed three times with ice-cold PBS. Protein concentrations were determined using the BCA assay (Sigma-Aldrich; Merck KGaA). Lysates were boiled in SDS loading buffer for $10 \mathrm{~min}$ after cleared by centrifugation $(12,000 \mathrm{x} \mathrm{g}, 10 \mathrm{~min}$, $\left.4^{\circ} \mathrm{C}\right)$. Immunoblotting was performed using $15 \%$ SDS-PAGE, transferred to a nitrocellulose membrane and detected using specific primary antibodies (LC3 and $\beta$-actin at $4^{\circ} \mathrm{C}$ overnight). The immunocomplexes were incubated with the appropriate fluorescein-conjugated horse anti-mouse $\operatorname{IgG}$ or the goat anti-rabbit $\operatorname{IgG}$ antibody ( $1 \mathrm{~h}$ at room temperature) and detected using ECL. In addition, $100 \mu \mathrm{g}$ protein was loaded 
A
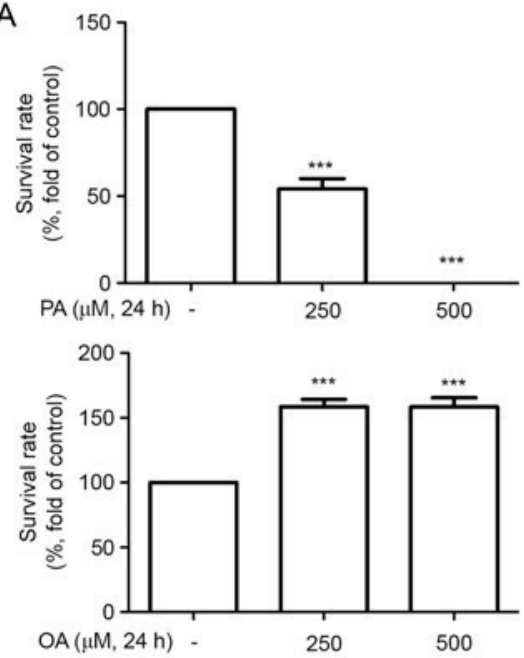

C
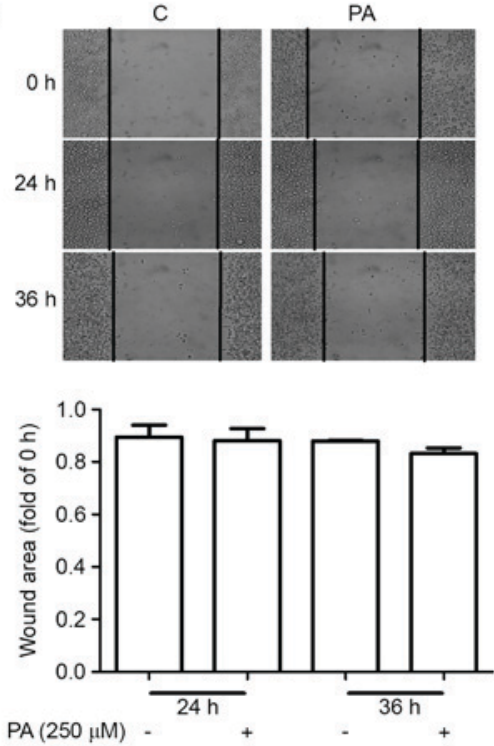

B

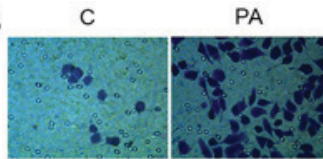

$\mathrm{OA}$
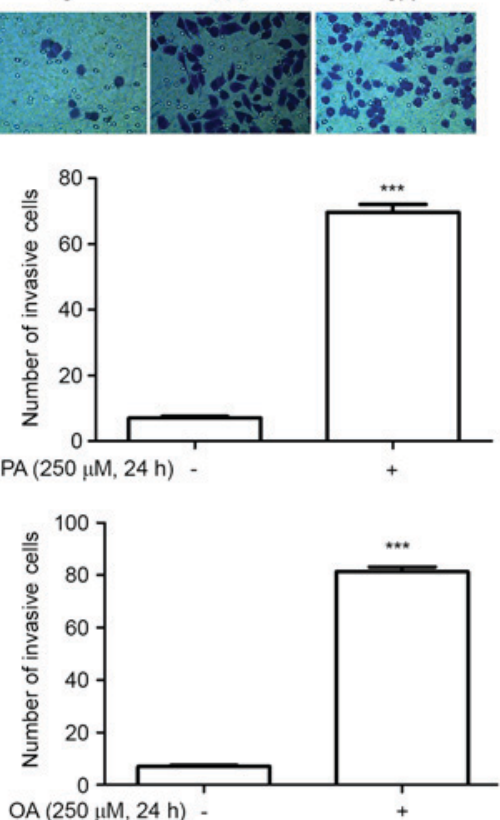

D
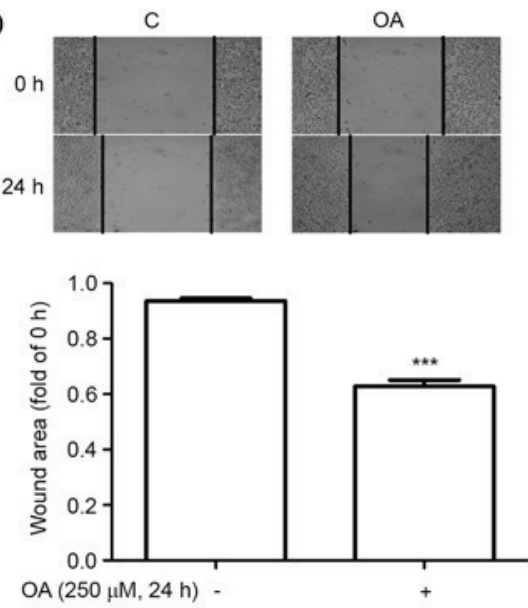

Figure 1. Effect of free fatty acids on the proliferation, migration and invasion of MDA-MB-231 cells. MDA-MB-231 cells were cultured with $0.5 \%$ BSA or BSA-bound PA or OA for 24 h. (A) Effect of PA and OA on cell proliferation. (B) Effect of PA and OA on cell invasion. (C) Effect of PA on wound healing. (D) Effect of OA on wound healing. ${ }^{* * *} \mathrm{P}<0.001$ vs. negative control. BSA, bovine serum albumin; PA, palmitate acid; OA, oleic acid; C, control.

per well. The ECL visualization reagent was purchased romm Cell Signaling Technology, Inc. and mixed fresh prior to use.

Statistical analysis. Results are expressed as the mean \pm standard error of the mean. The quantification of the relative increase in protein expression was performed using National Institutes of Health Scion Image software and was normalized with the control protein expression in each experiment. The values were representative of at least three independent experiments. Differences between mean values were examined using the paired Student's t-test. $\mathrm{P}<0.05$ was considered to indicate a statistically significant difference.

\section{Results}

Different effects of $P A$ and $O A$ on MDA-MB-231 cell growth. To investigate the effects of FFAs on cancer cell proliferation, MDA-MB-231 cells were incubated in $250 \mu \mathrm{M}$
BSA-bounded PA/OA for $24 \mathrm{~h}$. MTT and TUNEL assays were performed to measure cell proliferation and apoptosis. As demonstrated in Figs. 1 and 2, OA enhanced MDA-MB-231 cell proliferation while PA enhanced apoptosis. The effects of FFAs on MDA-MB-231 cell migration and invasion were then investigated using wound healing and Transwell assays. Mitomycin $\mathrm{C}$ was administered to inhibit cell proliferation, and it was determined that the relative wound area and the number of invading cells was significantly increased in cells that had been treated with OA for $24 \mathrm{~h}$. The invading cells were also significantly increased in cells treated with PA for $24 \mathrm{~h}$. However, no evident migration was observed in cells treated with PA for 24 or $36 \mathrm{~h}$.

Effects of FFAs on autophagy. As a specific marker for autophagy, LC3 is widely used to monitor autophagy. The present study detected autophagy through western blot analysis for LC3. When autophagy is induced, the cytosolic 

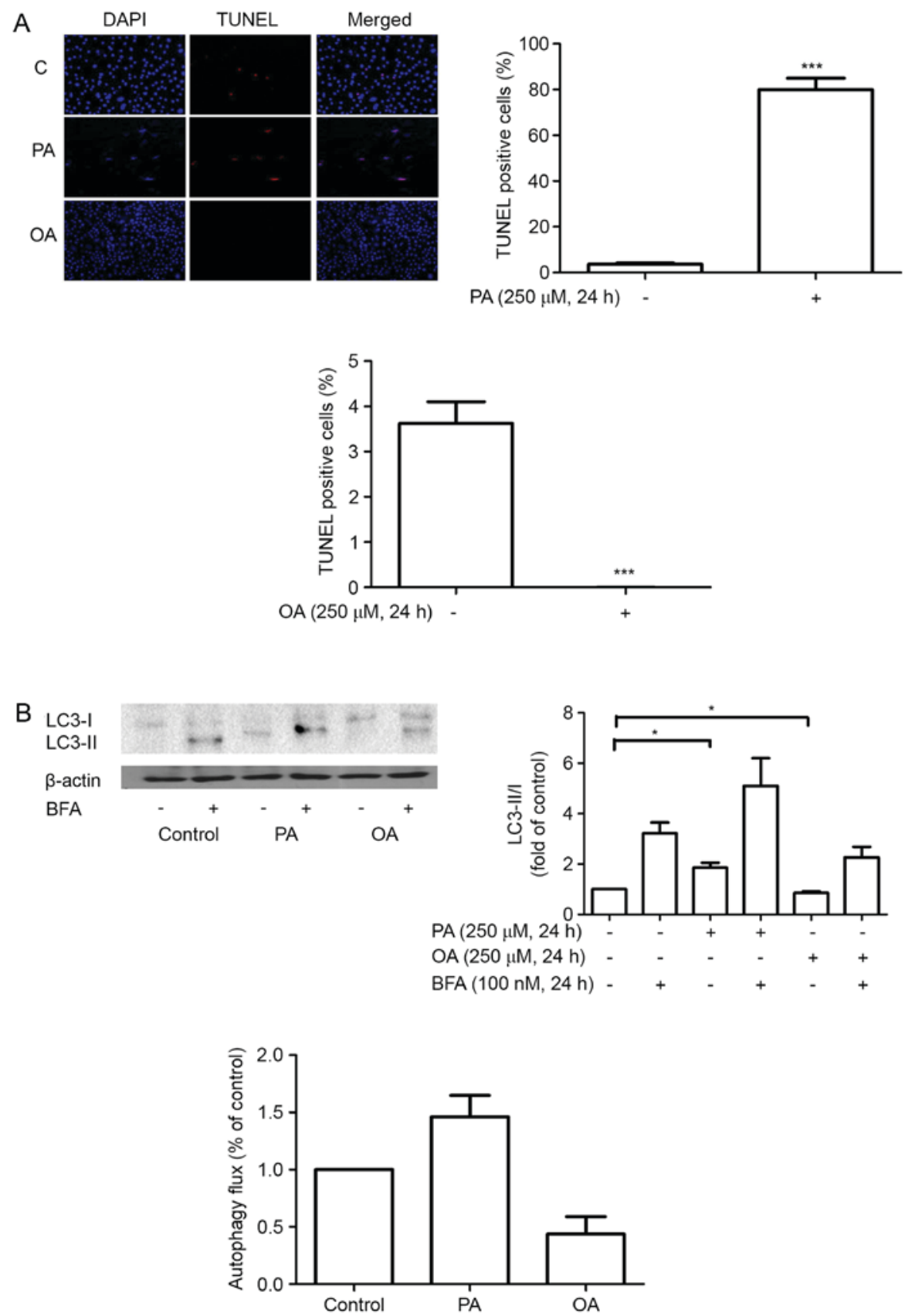

Figure 2. (A) Effect of PA and OA on MDA-MB-231 cell apoptosis. (B) Effect of free fatty acids on autophagy. The MDA-MB-231 cells were cultured with $0.5 \%$ BSA or $250 \mu \mathrm{M}$ BSA-bound PA or OA for $24 \mathrm{~h}$. The levels of LC3 and autophagy flux were determined by western blot analysis. A representative blot is shown. ${ }^{\mathrm{P}}<0.05$ and ${ }^{* * * *} \mathrm{P}<0.001$ vs. negative control. BSA, bovine serum albumin; PA, palmitate acid; OA, oleic acid; BFA, Bafliomycin A1; LC3, light chain 3.

form of LC3, LC3-I, is processed to the lipidated and autophagosome-associated form, LC3-II $(11,13)$. In the present study, treatment with PA led to an increased LC3II/I ratio in MDA-MB-231 cells, while treatment with OA led to a decrease. To conclusively establish the autophagy flux, LC3-II/I levels were determined using BFA, a type of inhibitor that inhibits the autolysosomal degradation step. The present result showed a rise of autophagy flux subsequent to treatment with PA and a concurrent decrease in OA (Fig. 2B). Therefore, the present study hypothesized that PA induced autophagy while OA inhibited autophagy in MDA-MB-231 cells.

Autophagy mediated the effect of FFAs on proliferation, migration and invasion in MDA-MB-231 cells. To confirm the functional consequences of autophagy on MDA-MB-231 cell growth, migration and invasion, MDA-MB-231 cells were incubated with autophagy inhibitor or inducer as well as PA or OA for $24 \mathrm{~h}$. It was confirmed that PA induced autophagy could be mitigated by 3-MA pretreatment (Fig. 3B) while OA-induced autophagy inhibition could be promoted by treatment with rapamycin (Fig. 3A). Cell growth, migration, and invasion were then assessed by MTT, TUNEL, wound healing, and Transwell assays, respectively. The results demonstrated that the autophagy inhibitor 3-MA significantly increased PA-induced cell proliferation (Fig. 4A), reduced the number of invading cells (Fig. 4B) and suppressed cell apoptosis (Fig. 5B). At the same time, autophagy inducer rapamycin significantly reduced OA induced cell proliferation (Fig. 4A), 
A

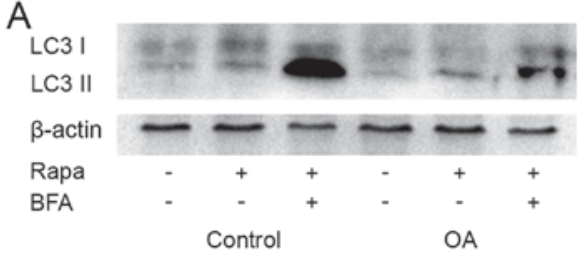

B

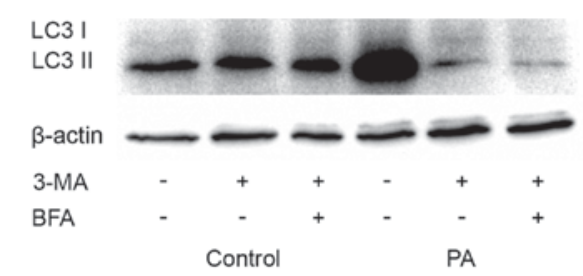

C

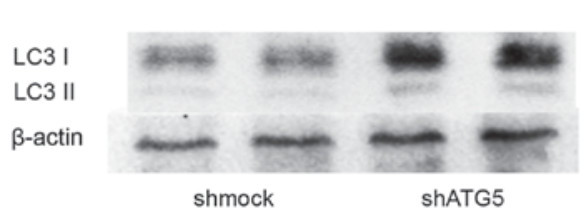

D

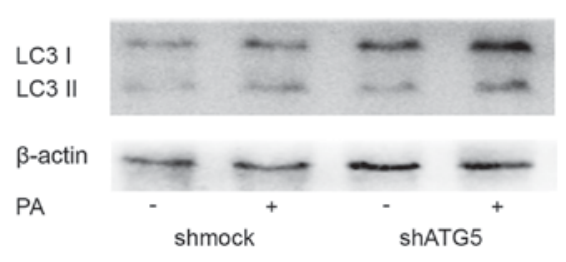

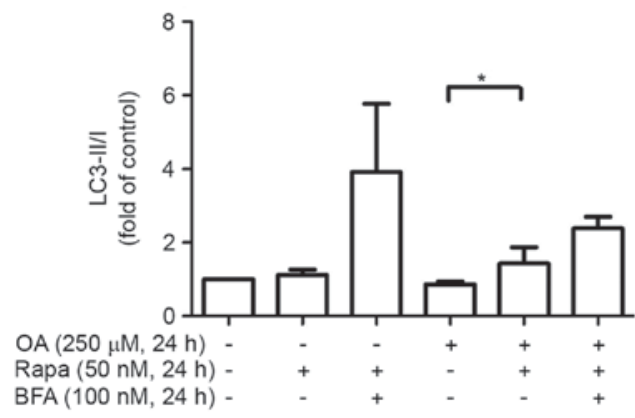
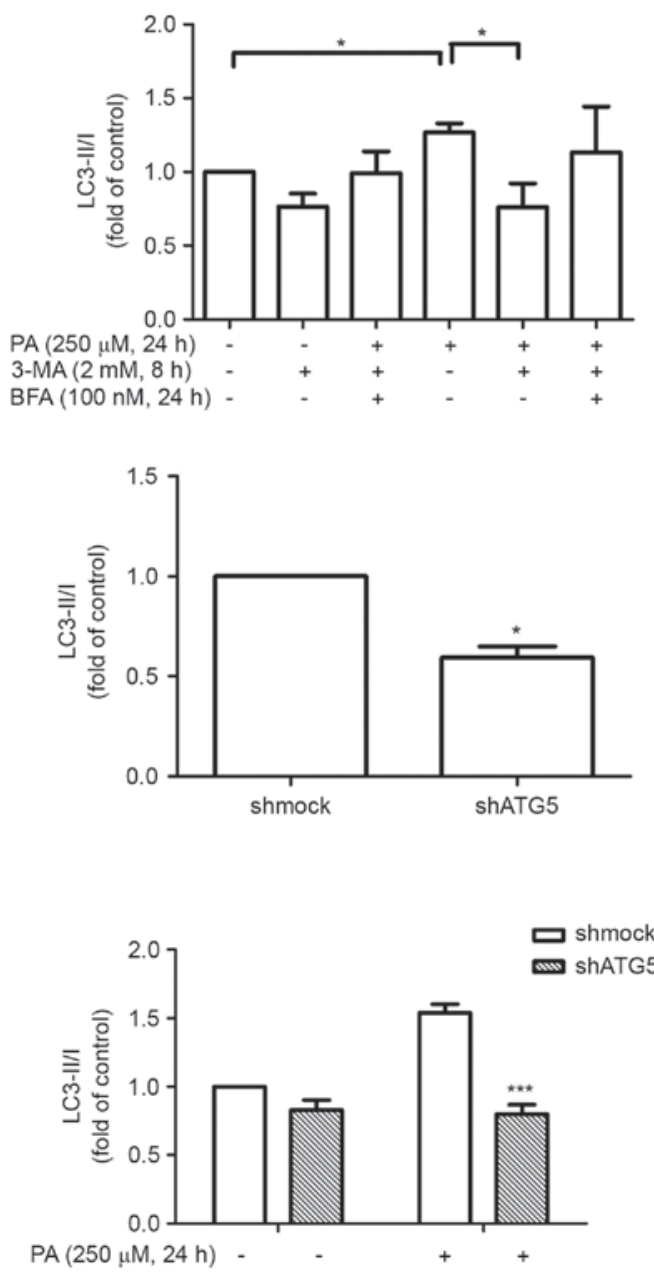

Figure 3. Effects of FFAs on autophagy. (A) The MDA-MB-231 cells were cultured with $250 \mu$ M BSA-bound OA and 50 nM Rapa for 24 h. (B) The MDA-MB-231 cells were pretreated with $2 \mathrm{mM} 3$-MA for $8 \mathrm{~h}$ and cultured with $250 \mu \mathrm{M}$ BSA-bound PA for $24 \mathrm{~h}$. (C) The cells transfected with shmock or shATG5 were detected by western blot analysis. (D) The cells transfected with shmock or shATG5 were treated with $250 \mu \mathrm{M}$ BSA-bound PA for 24 h. A representative blot is shown. ${ }^{*} \mathrm{P}<0.05$ and ${ }^{* * *} \mathrm{P}<0.001$ vs. negative control. Rapa, rapamycin; BSA, bovine serum albumin; PA, palmitate acid; OA, oleic acid; 3-MA, 3-methyladenine; shRNA, short hairpin RNA; shmock, control shRNA; shATG5, shRNA against autophagy protein 5; LC3, light chain 3.

reduced the number of invading cells (Fig. 4B) and suppressed wound closure (Fig. 5A).

shRNA against ATG5, which is known to initiate the formation of the autophagosome, was transfected into MDA-MB-231 cells. Western blot analysis confirmed that inhibition of ATG5 expression could attenuate PA-induced LC3-II accumulation in MDA-MB-231 cells (Fig. 3C and D). As depicted in Figs. 4A and B, and 5B, consistent with the exposure to 3-MA, silencing of ATG5 by treatment with PA caused a increase in cell proliferation, and decrease in cell apoptosis and invasion after $24 \mathrm{~h}$.

\section{Discussion}

Previous studies have reported accumulating evidence of metabolic reorganization during cancer development. Tumor cells gain a survival or growth advantage by adapting their metabolism to respond to environmental stress, the best-known aspect of which is the Warburg effect $(16,17)$. Understanding the metabolic differences between normal and tumor cells could provide the opportunity to design selective personalized therapy against breast cancer and other cancers (18). However, the metabolism of cancer cells is complex and involves various 

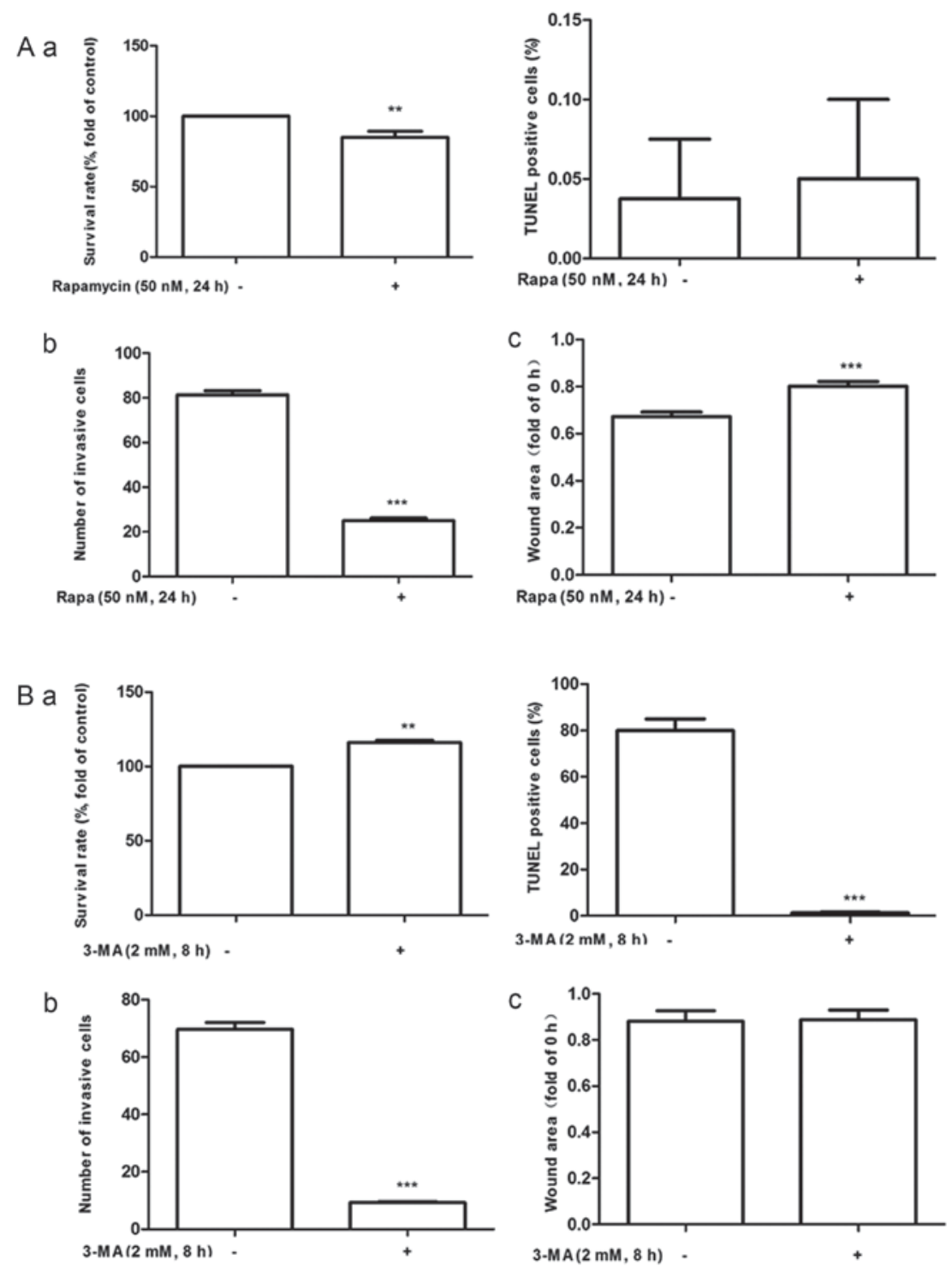

Figure 4. Autophagy-mediated effects of free fatty acids on MDA-MB-231 cell proliferation, migration and invasion. (A) The MDA-MB-231 cells were cultured with $250 \mu \mathrm{M} \mathrm{OA}$ and $50 \mathrm{nM}$ Rapa for $24 \mathrm{~h}$. We determined their difference on (a) proliferation, (b) invasion and (c) migration. (B) The MDA-MB-231 cells were pretreated with $2 \mathrm{mM} 3-\mathrm{MA}$ for $8 \mathrm{~h}$ and cultured with $250 \mu \mathrm{M}$ BSA-bound PA for $24 \mathrm{~h}$. The difference on (a) proliferation, (b) invasion and (c) migration were determined. ${ }^{* *} \mathrm{P}<0.01$ and ${ }^{* * *} \mathrm{P}<0.001$ vs. negative control. Rapa, rapamycin; $\mathrm{PA}$, palmitate acid; 3-MA, 3-methyladenine.
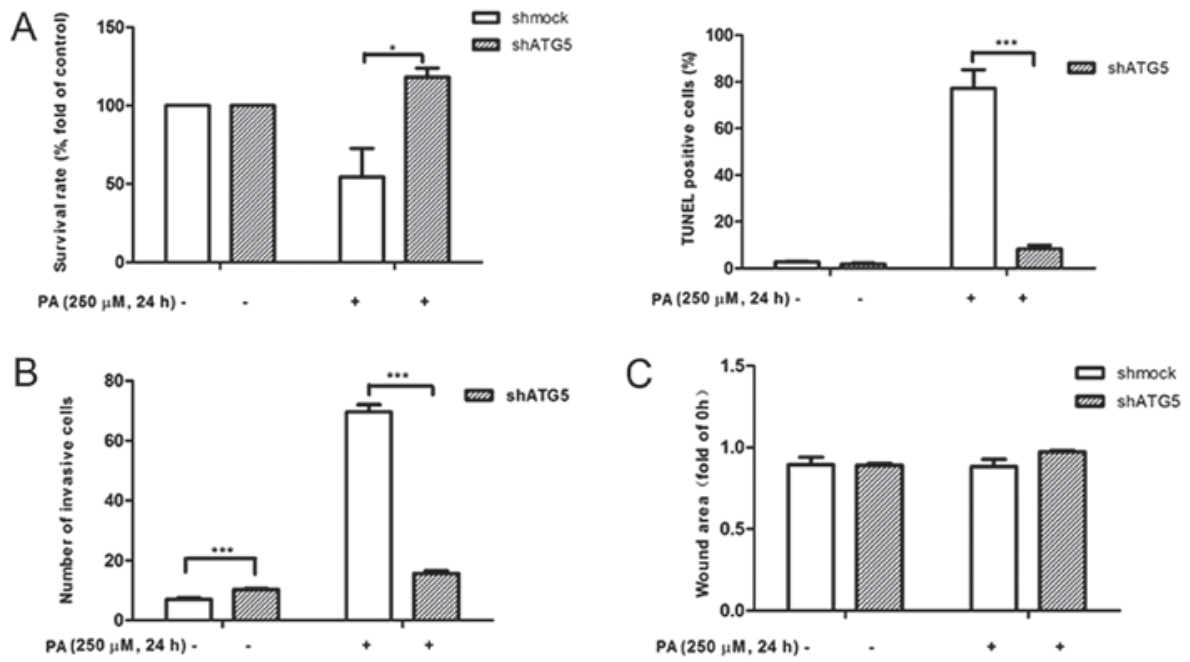

Figure 5. Autophagy-mediated effects of free fatty acids on MDA-MB-231 cell (A) proliferation, (B) invasion and (C) migration. The shATG5 and shmock cells were treated with $250 \mu \mathrm{M}$ PA. ${ }^{*} \mathrm{P}<0.05$ and ${ }^{* * * *} \mathrm{P}<0.001$ vs. negative control. PA, palmitate acid; shRNA, short hairpin RNA; shmock, control shRNA; shATG5, shRNA against autophagy protein 5 . 
rewiring of the metabolic pathways that occurs during malignant transformation. The data presented in the present study demonstrated that PA promotes MDA-MB-231 cell apoptosis and invasion while OA promotes proliferation, migration and invasion. Although FFA levels are generally elevated in breast cancer patients $(19,20)$, the role of FFA in health and disease in humans has been minimally investigated and understood.

It has been shown that autophagy may be induced by multiple stimuli, including nutrient deprivation, serum starvation, metabolic stress, radiation and anticancer drugs, in multiple cancer cells $(21,22)$. This has provided insight into the adaptation process for cells, which either allows tolerance of adverse conditions or triggers cell suicide mechanisms (23). Basal autophagy is essential to maintain cellular homeostasis and genomic integrity by degrading the aged or malfunctioning organelles and damaged or misfolded proteins $(11,24)$. It has been shown that autophagy is frequently upregulated in tumors in response to therapy and may protect the tumors during cancer development, but promote cell survival during cancer progression (22). However, the role of autophagy in cancer remains controversial, as it may suppress tumors during cancer development and promote cell survival during cancer progression (25).

In the present study, it was found that LC3-II accumulated in PA-treated MDA-MB-231 cells; this effect was enhanced in the presence of BFA and was blocked by 3-MA. Inhibition of ATG5 expression attenuated PA-induced LC3-II accumulation. The present results also determined that pretreatment with the autophagy inhibitor 3-MA significantly mitigated cell apoptosis and invasion, suggesting that high PA-induced MDA-MB-231 cell apoptosis and invasion are mediated by promoting autophagy. By contrast, LC3-II decreased in OA-treated MDA-MB-231 cells and was restored using rapamycin, which significantly reduced cell growth, migration and invasion, indicating that high OA-induced MDA-MB-231 cell proliferation, migration and invasion are mediated by inhibiting autophagy.

In summary, to the best of our knowledge, the present findings revealed that the difference between PA and OA-induced growth, migration and invasion of MDA-MB-231 cells is mediated by autophagy for the first time. This indicates the potential therapeutic importance of lipid regulation on human breast cancer.

\section{Acknowledgements}

The authors would like to thank Professor Lei Wei (Department of Pathology and Pathophysiology, Wuhan University School of Basal Medical School) for the gift of the MDA-MB-231 cell line.

\section{References}

1. American Cancer Society: Cancer Facts and Figures 2017 American Cancer Society, Atlanta, GA, 2017.

2. Zheng R, Zeng H, Zhang S, Chen T and Chen W: National estimates of cancer prevalence in China. Cancer Lett 370: 33-38, 2011.

3. Wolf I, Sadetzki S, Catane R, Karasik A and Kaufman B: Diabetes mellitus and breast cancer. Lancet Oncol 6: 103-111, 2005.
4. Nomura DK, Long JZ, Niessen S, Hoover HS, Ng SW and Cravatt BF: Monoacylglycerol lipase regulates a fatty acid network that promotes cancer pathogenesis. Cell 140: 49-61, 2010.

5. Przybytkowski E, Joly E, Nolan CJ, Hardy S, Francoeur AM, Langelier Y and Prentki M: Upregulation of cellular triacylglycerol-free fatty acid cycling by oleate is associated with long-term serum-free survival of human breast cancer cells. Biochem Cell Biol 85: 301-310, 2007.

6. Cantrill RC and Huang YS: Fatty acids and cancer. Nutrition 14: 235-237, 1998.

7. Patterson RE, Flatt SW, Newman VA, Natarajan L, Rock CL, Thomson CA, Caan BJ, Parker BA and Pierce JP: Marine fatty acid intake is associated with breast cancer prognosis. J Nutr 141: 201-206, 2011.

8. Ford JH: Saturated fatty acid metabolism is key link between cell division, cancer, and senescence in cellular and whole organism aging. Age (Dordr) 32: 231-237, 2010.

9. Patterson RE, Flatt SW, Newman VA, Natarajan L, Rock CL, Thomson CA, Caan BJ, Parker BA and Pierce JP: Marine fatty acid intake is associated with breast cancer prognosis. J Nutr 141: 201-206, 2011.

10. Abel S, Riedel S and Gelderblom WC: Dietary PUFA and cancer. Proc Nutr Soc 73: 361-367, 2014.

11. Rabinowitz JD and White E: Autophagy and metabolism. Science 330: 1344-1348, 2010.

12. Mizushima N, Levine B, Cuervo AM and Klionsky DJ: Autophagy fights disease through cellular self-digestion. Nature 451: 1069-1075, 2008.

13. Shi Z, Li CY, Zhao S, Yu Y, An N, Liu YX, Wu CF, Yue BS and Bao JK: A systems biology analysis of autophagy in cancer therapy. Cancer Lett 337: 149-160, 2013.

14. Zarzynska JM: The importance of autophagy regulation in breast cancer development and treatment. Biomed Res Int 2014: 710345, 2014.

15. Mizushima N, Levine B, Cuervo AM and Klionsky DJ: Autophagy fights disease through cellular self-digestion. Nature 451: 1069-1075, 2008.

16. Li S, Zhou T, Li C, Dai Z, Che D, Yao Y, Li L, Ma J, Yang X and Gao G: High metastaticgastric and breast cancer cells consume oleic acid in an AMPK dependent manner. PLoS One 9: e97330, 2014.

17. Kim S, Lee Y and Koo JS: Differential expression of lipid metabolism-related proteins in different breast cancer subtypes. PLoS One 10: e0119473, 2015.

18. DeBerardinis RJ and Thompson CB: Cellular metabolism and disease: What do metabolic outliers teach us? Cell 148: 1132-1144, 2012.

19. Lv ZH, Ma P, Luo W, Xiong H, Han L, Li SW, Zhou X and Tu JC: Association between serum free fatty acid levels and possible related factors in patients with type 2 diabetes mellitus and acute myocardial infarction. BMC Cardiovasc Disord 14: 159, 2014.

20. Lv W and Yang T: Identification of possible biomarkers for breast cancer from free fatty acid profiles determined by GC-MS and multivariate statistical analysis. Clin Biochem 45: 127-133, 2012.

21. Chen YR, Tsou B, Hu S, Ma H, Liu X, Yen Y and Ann DK: Autophagy induction causes a synthetic lethal sensitization to ribonucleotide reductase inhibition in breast cancer cells. Oncotarget 7: 1984-1999, 2016.

22. Huang YH, Yang PM, Chuah QY, Lee YJ, Hsieh YF, Peng CW and Chiu SJ: Autophagy promotes radiation-induced senescence but inhibits bystander effects in human breast cancer cells. Autophagy 10: 1212-1228, 2014.

23. Wang L, Yao L, Zheng YZ, Xu Q, Liu XP, Hu X, Wang P and Shao ZM: Expression of autophagy-related proteins ATG5 and FIP200 predicts favorable disease-free survival in patients with breast cancer. Biochem Biophys Res Commun 458: 816-822, 2015.

24. Eng $\mathrm{CH}$ and Abraham RT: The autophagy conundrum in cancer: Influence of tumorigenic metabolic reprogramming. Oncogene 30: 4687-4696, 2011.

25. Boyer-Guittaut M,Poillet L,Liang Q, Bôle-Richard E, Ouyang X, Benavides GA, Chakrama FZ, Fraichard A, Darley-Usmar VM, Despouy G, et al: The role of GABARAPL1/GEC1 in autophagic flux and mitochondrial quality control in MDA-MB-436 breast cancer cells. Autophagy 10: 986-1003, 2014. 